\title{
POLÍTICAS DE ORDENACIÓN TERRITORIAL Y URBANÍSTICA EN LOS ESPACIOS COSTEROS ANDALUCES.
}

\author{
FEDERICo BenJamín Galacho JimÉneZ
}

\begin{abstract}
RESUMEN
El paisaje costero está afectado por la concentración de numerosas actividades en una escasa superficie y por la presencia de importantes continuos urbanizados. Esta situación provoca alteraciones irreversibles, fundamentalmente en los ámbitos urbanos, en las áreas turísticas y en las zonas especializadas en torno a la agricultura intensiva. De especial trascendencia será en un futuro la aplicación real de políticas de ordenación territorial y urbanística orientadas hacia la disminución de la presión urbana y de infraestructuras en el litoral, así como hacia su regeneración, recuperación y acondicionamiento para uso y disfrute público. En este texto, se exponen los marcos legislativos y criterios de ordenación territorial y urbanística a distintos niveles: comunitario, nacional, autonómico y local.
\end{abstract}

\section{ABSTRACT}

The coastal landscape is affected by the concentration of numerous activities in a scarce surface and by the presence of important continuous urbanized. This situation causes irreversible alterations, fundamentally in the urban environments, in the tourist areas and in the zones of specialized around the intensive agriculture. Of special significance will be in a future the real application of politics of urban development and territorial ordering oriented toward the decrease of the urban pressure and of infrastructures in the seaboard, as well as toward its regeneration, recovery and conditioning for use and public enjoyment. In this text, the legislative frameworks they are exposed and criteria of urban development and territorial ordering to different levels: European Common, national, autonomous and local spaces.

\section{INTRODUCCIÓN}

Un aspecto fundamental de la lógica de ocupación del suelo de los espacios costeros andaluces ha sido el desarrollo mal planificado y, en muchos 
momentos, descontrolado de las actividades económicas predominantes. El turismo y la construcción se han venido desarrollando de forma espontánea, sin planificación, lo que ha provocado graves problemas al medio ambiente, sustento fundamental de las actividades mismas. Los impactos negativos generados por un modelo de desarrollo económico insostenible como el que se está llevando a cabo, no sólo están revertiendo sobre el territorio y la calidad de vida de los ciudadanos sino también en el desenvolvimiento normal de las actividades económicas mismas. El desafío actual parte de relacionar planificación y desarrollo, bajo perspectivas de sostenibilidad ambiental, solucionando los problemas presentados por el modelo económico desarrollado en los últimos cuarenta años y que requiere ser reinventado en su estructura, de modo que no se vuelva a producir la repetición de esquemas ya obsoletos e insostenibles.

Un aspecto crucial son las formas de ocupación territorial y la configuración del sistema de asentamientos. Éstas se han basado fundamentalmente en el uso indiscriminado del espacio. Si realizamos una caracterización morfológica del mismo podemos observar como los centros urbanos tradicionales, con centralidad establecida en sus cascos históricos, se constituyen en epicentros de desarrollo urbano sólo de modo parcial, extendiéndose la trama urbana de modo disperso por el territorio en relación a los ejes viarios. Al mismo tiempo, la vivienda se constituye en factor fundamental de movilidad y relocalización de la población, nacional y/o extranjera, hecho que introduce especial importancia a su distribución territorial y por derivación, como factor imprescindible a considerar, de cara a la mejora del nivel de articulación entre los diferentes núcleos y espacios urbanos. No obstante, otras actividades pugnan por el espacio con el desarrollo turístico, los cultivos agrícolas intensivos y los usos urbanos no derivados directamente de la actividad turística.

La expansión urbana en el litoral es, por lo que se refiere al urbanismo, el aspecto más acuciante. El espacio urbanizado se extiende invadiendo zonas naturales y rurales a un ritmo más rápido que el crecimiento de la población (20\% de expansión en los últimos 20 años, con sólo un $6 \%$ de la población). Se están sustituyendo espacios verdes (espacios naturales y agrícolas de gran valor) por zonas destinadas a complejos de ocio que incluyen viviendas de baja densidad y campos de golf, como la propuesta con más aceptación en la actualidad.

El modelo de expansión urbana desarrollado refuerza la necesidad de desplazamientos y la dependencia del automóvil, aumentando así la congestión del tráfico, el consumo de energía y las emisiones contaminantes. Este hecho conlleva la necesidad de construcción de más infraestructuras viarias, pasando a ser su localización otro acuciante problema relacionado con el uso del suelo. Los distintos municipios litorales compiten entre sí por atraer las inversiones 
y ofrecen incentivos y facilidades, entre otras la reclasificación del suelo necesario para proyectos residenciales, deportivos o comerciales. El aumento de la movilidad ha dado lugar a la aparición de nuevos modelos de urbanismo, en los que las zonas ya urbanizadas cuentan con diferentes "centros", cada uno de los cuales puede estar especializado en una función (comercios, ocio, residencias) y entrar en competencia con los demás. Al mismo tiempo, aparecen vínculos entre zonas urbanas contiguas, produciéndose redes de ciudades (Bahía de Cádiz, Bahía de Algeciras, Costa del Sol Occidental o aglomeración urbana de Málaga).

La regulación de la expansión urbana ilimitada debería ser una cuestión prioritaria para todas las regiones, y para las costeras aún más. Las estrategias y políticas de ordenación territorial para las zonas urbanas emergentes deben coordinarse para que coincidan a nivel regional, nacional, comunitario y sobre todo, local, de modo que pueda ser relativamente factible establecer estrategias para un medio ambiente urbano sano y de alta calidad. La integración vertical en los distintos niveles competenciales es vital.

Otro aspecto de especial trascendencia es la aplicación real de políticas orientadas hacia la disminución de la presión urbana y de infraestructuras en el litoral, así como hacia su regeneración, recuperación y acondicionamiento para uso y disfrute público. De esta forma, la planificación urbanística y territorial debe considerar el espacio litoral como notablemente sobreutilizado, orientado sus determinaciones en consonancia con tales circunstancias. El uso del suelo es un factor fundamental en este sentido. La mala gestión y utilización del suelo está creando zonas urbanas en el litoral poco atractivas para vivir y está produciendo formas de asentamiento no sostenibles.

\section{CRITERIOS EMANADOS DE LA UNIÓN EUROPEA}

Durante los últimos años, las políticas comunitarias han mostrado un especial interés por las regiones del litoral europeo. Así, los responsables políticos de la Unión Europea han decidido crear una política coordinada para las zonas costeras (COM: 2000, 547 de 27.9.2000). Además de las medidas dirigidas a mejorar las políticas que afectan a las zonas costeras, la Comisión Europea ha venido instando a los Estados miembros a que establezcan estrategias nacionales sobre lo que se ha llamado "gestión integrada de las zonas costeras" (UE: 2001).

Desde la Comisión se pretende promocionar esa gestión integrada reuniendo a los distintos agentes y políticas que a nivel local, regional, nacional y europeo actúan sobre las regiones costeras de la Unión. Parece que se ve 
claro que sin una coordinación a todos los niveles, cualquier propuesta que se realice para proteger el litoral será prácticamente en vano. Uno de los principios fundamentales para una política eficaz de gestión integrada es encarar los problemas de las zonas costeras en un contexto lo más amplio posible. En el pasado, muchas medidas adoptadas con las mejores intenciones para mejorar el estado de los espacios costeros se han visto abocadas al fracaso porque los problemas se consideraron como si fueran independientes. La cuestión del turismo en las regiones costeras, por ejemplo, no puede abordarse de una manera eficaz si no se tienen en cuenta también otros muchos factores como pueden ser los recursos hídricos, los usos del suelo o el impacto del turismo sobre los hábitats naturales existentes, etc.

La gestión integrada de las zonas costeras se centrará en establecer estrategias de planificación y gestión de los recursos y el espacio litoral porque es más que una mera medida medioambiental, aunque el objetivo fundamental de la propuesta consiste en proteger el funcionamiento de los ecosistemas naturales, la gestión integrada aboga también interconectar las actividades económicas al objetivo mencionado; con lo que se espera conseguir el aumento del bienestar social de la población de estos espacios.

Los días 18 y 19 de abril de 2002 tuvo lugar en La Vila Joiosa (Alicante) el Primer Foro Europeo sobre Estrategias Comunitarias para la Gestión Integrada de Zonas Costeras. Este Foro, que representaba la primera reunión de máximos responsables de Costas de la Unión Europea, con asistencia también de los directores generales de Costas de los países Candidatos, en su día, a la Ampliación de la UE, partió de un principio de acuerdo del Consejo de la UE y del Parlamento Europeo, inmediatamente anterior, sobre una recomendación cuyo objetivo era fomentar la gestión integrada de las zonas costeras de Europa. Los problemas ambientales en general, del urbanismo en zonas costeras y del riesgo de inundaciones ocuparon lugar destacado entre los temas tratados. De acuerdo con lo acordado en este Primer Foro, nuestro país recibiría el encargo de liderar la puesta en marcha de Estrategias Nacionales para coordinar todas las actividades que se desarrollen en el litoral europeo, dentro de políticas que deberán enmarcarse en la Estrategia Europea para el Desarrollo Sostenible y el VI Programa de Acción en materia de Medio Ambiente.

Con la directiva comunitaria 2000/60/CE ${ }^{1}$ sobre calidad del agua (directiva marco sobre el agua) se abordaban los problemas de contaminación del

1. Directiva 2000/60/CE del Parlamento Europeo y del Consejo, de 23 de octubre de 2000, por la que se establece un marco comunitario de actuación en el ámbito de la política de aguas [Diario Oficial L 327 de 22.12.2000]. Modificado por el siguiente acto: Decisión $n^{\circ}$ 2455/2001/CE del Parlamento Europeo y del Consejo, de 20 de noviembre de 2001 [DO L 331 de 15.12.2001]. 
litoral basándose en el sentido común pero también con ánimo innovador y tomando cada una de las cuencas hidrográficas como punto de partida para la protección de los recursos hídricos. En relación con las zonas costeras, la directiva marco sobre el agua debía obligar a los Estados miembros a adoptar medidas coherentes para combatir todas las fuentes de contaminación, sean de origen terrestre o marino. La legislación comunitaria concede a los gobiernos un plazo de 15 años (a contar desde el año 2000) para conseguir un buen grado de calidad de las aguas costeras mediante la aplicación de medidas coherentes basadas en la gestión de las cuencas fluviales.

Con fecha 11 de febrero de 2004, la Comisión de las Comunidades Europeas publicó la Comunicación (COM (2004)60 final)² titulada: "Hacia una estrategia temática sobre el medio ambiente urbano". La presente Comunicación es la primera fase de la preparación de la Estrategia final, que ha sido lanzada en el verano de 2005. Las ideas y enfoques iniciales que se presentaron fueron el resultado de consultas realizadas con ciudades y otras partes interesadas, grupos de trabajo de expertos independientes y grupo de expertos de la UE sobre medio ambiente urbano. Las contribuciones de estos grupos pueden consultarse en la dirección de Internet de la Comisión en materia de medio ambiente urbano ${ }^{3}$.

Si bien en la Comunicación mencionada no se hace referencia explicita al medio litoral, entendemos nosotros que dada la configuración urbana de éste en amplios espacios del litoral andaluz, podemos referenciar este documento al ámbito que estamos analizando. Nos interesa destacar las consideraciones que dicho documento hace sobre el medio urbano, principalmente en lo que se refiere a que la ausencia de atención a las implicaciones medioambientales que comportan las decisiones políticas y la ausencia de planificación sistemática del entorno urbano para garantizar su calidad son las razones que explican la situación actual, con graves consecuencias tanto para el medio ambiente como para la economía del medio urbano y sus ciudadanos. En la actualidad los agentes económicos y la mayoría de las administraciones locales están muy lejos de aceptar la necesidad de una planificación en pro de un alto nivel de protección medioambiental ya que entienden que esto supondría un freno a la obtención del beneficio económico de sus actividades para los primeros y al desarrollo de sus municipios para los segundos. Sin embargo, la planificación en una condición sine qua non para fomentar el pretendido desarrollo sostenible de las ciudades y garantizar un alto nivel de calidad de vida a los ciudadanos que habitan este medio.

2. Comunicación de la Comisión al Consejo, al Parlamento Europeo, al Comité Económico y Social Europeo y al Comité de las Regiones. Bruselas, 11.02.2004

3. www.europa.eu.int/environment/urban/thematic_strategy.htm 
La estrategia de la UE sobre el medio ambiento urbano constituye un paso importante hacia la consecución del objetivo anteriormente mencionado, y se fundamenta en una serie de iniciativas que han contribuido al desarrollo de la política europea sobre el medio urbano. Esta estrategia forma parte del Sexto Programa de Acción de la Comunidad Europea en materia de Medio Ambiente denominado "Medio Ambiente 2010: el futuro en nuestras manos", siendo una de las siete estrategias temáticas del Programa, introducidas para conferir un enfoque globalizador a importantes aspectos medioambientales que se caracterizan por su complejidad, el gran número de agentes implicados y la necesidad de soluciones múltiples e innovadoras. En definitiva el objetivo general de esta estrategia sobre el medio ambiente urbano: "es mejorar los resultados medioambientales y la calidad del entorno en las zonas urbanas, y garantizar un medio de vida sano para los ciudadanos europeos, reforzando la contribución del medio ambiente al desarrollo urbano sostenible" (COM: 2004-60 final, 5).

Con el fin de cumplir el mandato recogido en el Sexto Programa de Acción de la Comunidad Europea en materia de Medio Ambiente, la estrategia temática sobre el medio ambiente urbano se ha venido centrando en cuatro temas trasversales, fundamentales para la sostenibilidad de las ciudades a largo plazo, que entroncan de forma clara con los grandes pilares económicos y sociales del desarrollo sostenible, y que son los que más posibilidades pudieran tener de lograr avances significativos: la gestión urbana sostenible, el transporte urbano sostenible, la construcción sostenible y el urbanismo sostenible. Los temas se presentan de forma separada pero existen entre ellos interacciones significativas (COM 2004-60 final).

Las actuales iniciativas comunitarias en materia de urbanismo se dirigen a que la planificación urbanística consiga modelos de asentamiento urbano sostenible y tengan en cuenta los riesgos medioambientales. Existen varias directivas que pretenden ejercer influencia sobre el uso del suelo urbano, entre ellas las Directivas sobre la evaluación de impacto medioambiental ${ }^{5}$ y sobre la evaluación medioambiental estratégica ${ }^{6}$, así como la Directiva marco del

4. Decisión n ${ }^{\circ}$ 1600/2002/CE del Parlamento Europeo y del Consejo, de 22 de julio de 2002, por lo que se establece el Sexto Programa de Acción Comunitario en Materia de Medio Ambiente (DO n ${ }^{\circ}$ L 242 de 10.9.2002, p. 1).

5. Directiva 85/337/CEE del Consejo, de 27 de junio de 1985, relativa a la evaluación de las repercusiones de determinados proyectos públicos y privados sobre el medio ambiente, modificada por la Directiva 97/11/CE.

6. Directiva 2001/42/CE del Parlamento Europeo y del Consejo, de 27 de junio de 2001, relativa a la evaluación de los efectos de determinados planes y programas en el medio ambiente. 
agua ya mencionada ${ }^{7}$. Por otra parte, la Perspectiva Europea de Ordenación Territorial (PEOT), elaborada en 1999 por el Comité de Desarrollo Territorial, ha sido adoptada por todos los Estados miembros con carácter voluntario. En ella se establecen los objetivos y orientaciones para un desarrollo territorial equilibrado y sostenible; un tercio de las 60 opciones políticas acordadas abordan directamente la cuestión de cómo controlar la expansión física del proceso urbano (PEOT, 1999). A partir de esta iniciativa, el Observatorio en red de la ordenación del territorio europeo (ESPON) efectúa y coordina la investigación en el ámbito de la planificación territorial, y está instaurando un marco de recogida de datos espaciales para la creación de una Infraestructura de Información Espacial en Europa (INSPIRE) ${ }^{8}$, que deberá proporcionar información precisa para la formulación, puesta en marcha, seguimiento y evaluación de las políticas comunitarias en materia de urbanismo y ordenación del territorio.

Las normas que han regido los Fondos Estructurales y las Directrices para el período $2000-2006^{9}$ establecían la necesidad de adoptar enfoques sostenibles para el uso del suelo urbano. Por ejemplo, dando prioridad a la rehabilitación de emplazamientos industriales abandonados por encima de la creación de nuevos polos industriales. En esta línea se encuentra la iniciativa URBAN II que sostiene la utilización mixta y no perjudicial para el medio ambiente a la hora de reutilizar emplazamientos industriales abandonados, disminuyendo la presión ejercida por la creación de nuevos polos industriales y la expansión urbana.

En todos los documentos comunitarios sobre el medio ambiente urbano predomina el modelo de los asentamientos con usos múltiples con recuperación de terrenos industriales abandonados y los solares vacíos o espacios vacantes, donde la expansión urbana tiene lugar de manera planificada en vez de improvisada, contrariamente a como se está desarrollando el proceso urbano a nivel local en muchos municipios del litoral andaluz. La estrategia planteada en los documentos comunitarios apoya el modelo mencionado para los espacios urbanos europeos reconociendo, al mismo tiempo, que existen límites a la densidad de población, y exponiendo que en muchos casos prevalece la tendencia a olvidar que algunas zonas urbanas han generado un entorno de mala calidad debido a la superpoblación, y entendiendo también, que reconvertir zonas para usos múltiples es más fácil que invertir la tendencia a la expansión urbana incontrolada o aumentar la densidad de la ocupación del suelo.

7. Directiva 2000/60/EC.

8. www.ec-gis.org/inspire

9. Comunicación de la Comisión sobre los Fondos Estructurales y su coordinación con el Fondo de Cohesión-Directrices para los programas del período 2000-2006 (DO C 267, 22.9.1999). 
Se entiende que la Unión Europea no puede fijar un sistema uniforme para adoptar decisiones sobre ordenación del territorio, ni definir el modelo de asentamiento urbano "ideal", ya que cada espacio geográfico es único y las soluciones para llegar a un medio ambiente urbano sostenible son específicas de cada uno de ellos. No obstante, parece estar claro que si atendemos al caso del litoral comunitario y concretamente al andaluz, la mayoría de los enfoques que se están llevando a cabo son insostenibles y las estrategias que emanen desde la instancia comunitaria debería procurar impedirlos y obligar a los Estados miembros a promover otras alternativas más sostenibles. De hecho, las acciones que se proponen en los documentos que hemos seleccionado a lo largo de este apartado persiguen la gestión urbana sostenible. También se considera muy importante que la Comisión elabore Directrices sobre cuestiones concretas que podrían tener una influencia positiva sobre la práctica diaria y que, por ejemplo, podrían consistir en Directrices sobre la ubicación y la densidad de los nuevos proyectos inmobiliarios, la integración de los espacios naturales, la renovación de las zonas urbanas para mejorar sus sostenibilidad, la continuidad del tejido urbano (conexión de los tejidos urbanos nuevos y antiguos) o el establecimiento de medidas que dificulten la construcción en la áreas no urbanizadas.

\section{CRITERIOS EMANADOS DEL GOBIERNO CENTRAL}

Para frenar la situación de degradación del litoral español, que en muchos casos ha sido irreversible, y en cumplimiento del mandato constitucional previsto en el artículo $132.1^{10}$, el Gobierno Central ha arbitrado diversos instrumentos de planificación. Destacamos la aprobación de la Ley de Costas (Ley 22/1998, de 28 de Julio) que recoge tanto los criterios contenidos en la Recomendación 29/1973, del Consejo de Europa, sobre protección de zonas costeras, como en la Carta del Litoral de 1981, de la UE y en otros planes y programas de la misma. Dicha Ley ha sido desarrollada mediante el Reglamento, aprobado por Real Decreto 1471/1989 de 1 de diciembre de 1989, (BOE 12.12.1989; corrección de errores en BOE 23.01.1990). Dicho Reglamento ha sido modificado parcialmente por el Real Decreto 1112/1991,

10. La Constitución Española de 1978 establece en su artículo 132.1 que la ley ha de regular el régimen jurídico del dominio público, inspirándose en los principios de inembargabilidad, inalienabilidad e imprescriptibilidad. En este contexto, es evidente la relevancia del Dominio Público marítimo-terrestre, pues es el único de cuantos existen directamente individualizado y definido como tal en la Constitución (art. 132.2), probablemente con la finalidad de acabar con las anteriores confusiones y actitudes contrarias a la demanialidad de un espacio tan importante como el litoral. 
de 18 de septiembre (BOE del 6 de octubre), a raíz de dos sentencias del Tribunal Constitucional ${ }^{11}$. Recientemente, la Ley de Costas ha sido modificada, en siete de sus artículos, mediante la Ley 53/2002, de 30 de diciembre, de Medidas Fiscales, Administrativas y del Orden Social (BOE núm. 313, de 31.12.2002) ${ }^{12}$.

Basados en la Ley de Costas y en las modificaciones posteriores, el Gobierno Central ha desarrollado una serie de actuaciones en materia de política litoral. Los dos principales objetivos desarrollados a través de la Dirección General de Costas, del Ministerio de Medio Ambiente han sido la recuperación de las zonas degradadas y preservación de los ecosistemas costeros frente a las distintas amenazas, mediante las actuaciones en la costa y la recuperación del dominio público marítimo-terrestre respecto de las zonas ocupadas ilegalmente, mediante la realización de deslindes pertinentes de la costa, necesarios para la correcta aplicación de la primera norma mencionada y en cumplimiento de los establecido en el artículo 11 de la misma.

Respecto al primer objetivo mencionado, la mayor parte de las actuaciones concretadas y realizadas por el Ministerio de Medio Ambiente en las costas españolas ha consistido en la creación de senderos, en la reutilización de dunas, en la construcción y el acondicionamiento de paseos marítimos y en la rehabilitación de playas. De este modo, como actuaciones emblemáticas podemos citar las siguientes, circunscritas al litoral andaluz (MMA: 2004): la regeneración y conservación de los sistemas dunares de la Flecha Litoral de El Rompido, Lepe (Huelva), la restauración de las marismas de la Algaida, en Sánlucar de Barrameda (Cádiz), con retirada de los rellenos realizados por el puerto de Sevilla, la restauración del hábitat del camaleón en Chipiona (Cádiz), la recuperación de las playas de Guadalquitón y Campo Soto en el término municipal de San Roque (Cádiz) y el Plan de Actuaciones en la costa de Málaga, presentado en mayo de 2002 con un contenido de 68 actuaciones, centradas en medidas de recuperación medioambiental, mejora de los accesos, regeneración y acondicionamiento de playas y lucha contra la erosión.

Paralelamente se ha promovido la continuación de un plan de deslindes con la finalidad de dar cumplimiento a las directrices comunitarias expues-

11. En efecto, el Tribunal Constitucional dictó las sentencias 149/1991, de 4 de julio, y 198/1991, de 17 de octubre, en los recursos de inconstitucionalidad interpuestos contra determinados preceptos de la Ley de Costas, y en los conflictos de competencias planteados en relación con otros del Reglamento General para desarrollo y ejecución de la Ley de Costas.

12. Las modificaciones de la citada Ley de Medidas, contenidas en el artículo 120 de la misma, tienen por objeto coordinar las actuaciones de las Administraciones con competencias concurrentes en el ámbito costero, teniendo en cuenta los postulados del Tribunal Constitucional, localizados en las sentencias mencionadas anteriormente, a los efectos de delimitar las competencias de las diferentes Administraciones actuantes. 
tas anteriormente con referencia a la pretendida implantación de una política costera basada en una visión integral del litoral y orientada al logro de un desarrollo acorde a las cualidades y características del medio. Sin embargo, esta iniciativa se ha topado con un marco de escaso consenso entre las administraciones implicadas y, en estos casos, se han llevado a cabo actuaciones que han tenido el efecto contrario a lo pretendido, como por ejemplo, la ocupación flagrante del dominio público o el incumplimiento manifiesto de la norma con proyectos de actuaciones poco acordes con criterios respetuosos con los ecosistemas litorales. El caso es que la Ley de Costas ha repartido las competencias en materia de gestión del litoral entre las distintas Administraciones Públicas, y con ello las servidumbres legales previstas en la Ley de Costas para la protección del dominio público marítimo-terrestre ${ }^{13}$. De esto modo, las cuestiones urbanísticas de las zonas de servidumbre son competencia de los Ayuntamientos, de conformidad con el planeamiento vigente y sin perjuicio de las competencias de las Comunidades Autónomas en la aprobación de los correspondientes planes de ordenación y las autorizaciones en la zona de servidumbre de protección. Atribuidas inicialmente en la Ley de Costas y el Reglamento a la Administración General del Estado, han pasado a las Comunidades Autónomas ${ }^{14}$, pero sin perjuicio de las competencias de los Ayuntamientos afectados para otorgar las licencias urbanísticas, en su caso.

Esta política de deslindes no ha impedido en que se sigan produciéndose actuaciones que atentan contra el uso público del dominio público marítimoterrestre, como es el caso que muestra la figura núm. 1.

A pesar de todo, se considera necesaria la incorporación de enclaves de gran valor ecológico al dominio público marítimo-terrestre con el objetivo de aumentar su protección ambiental y potenciar el entorno natural y paisajístico de cada zona, o en su caso, quede asegurada su protección. Como ejemplo de esta situación podemos citar el caso de las Dunas de Artola (Marbella),

13. La Ley de Costas contempla y regula las siguientes servidumbres:

A)Una zona de servidumbre de protección de 100 metros, ampliable hasta 200 metros. 20 metros en suelo urbano clasificado como tal a la entrada en vigor de la Ley de Costas, que se extiende a lo largo de la costa tierra adentro desde la ribera del mar y en la que se sitúan los servicios y equipamientos públicos.

B) Una zona de servidumbre de tránsito de 6 metros, ampliable hasta 20 metros, desde la ribera del mar, y que, por tanto, queda integrada en la zona de protección.

C) Una zona de influencia, de un mínimo de 500 metros desde la ribera del mar y en la que se establecen condiciones mínimas para la protección del dominio público marítimoterrestre, que deberán ser respetadas por la ordenación territorial y urbanística.

14. Sentencia del Tribunal Constitucional 149/1991, declarando inconstitucional el artículo 110. b) de la Ley de Costas y S.T.C. 198/1991, declarando también inconstitucional el apartado 1.b) del artículo 203 del RC). 


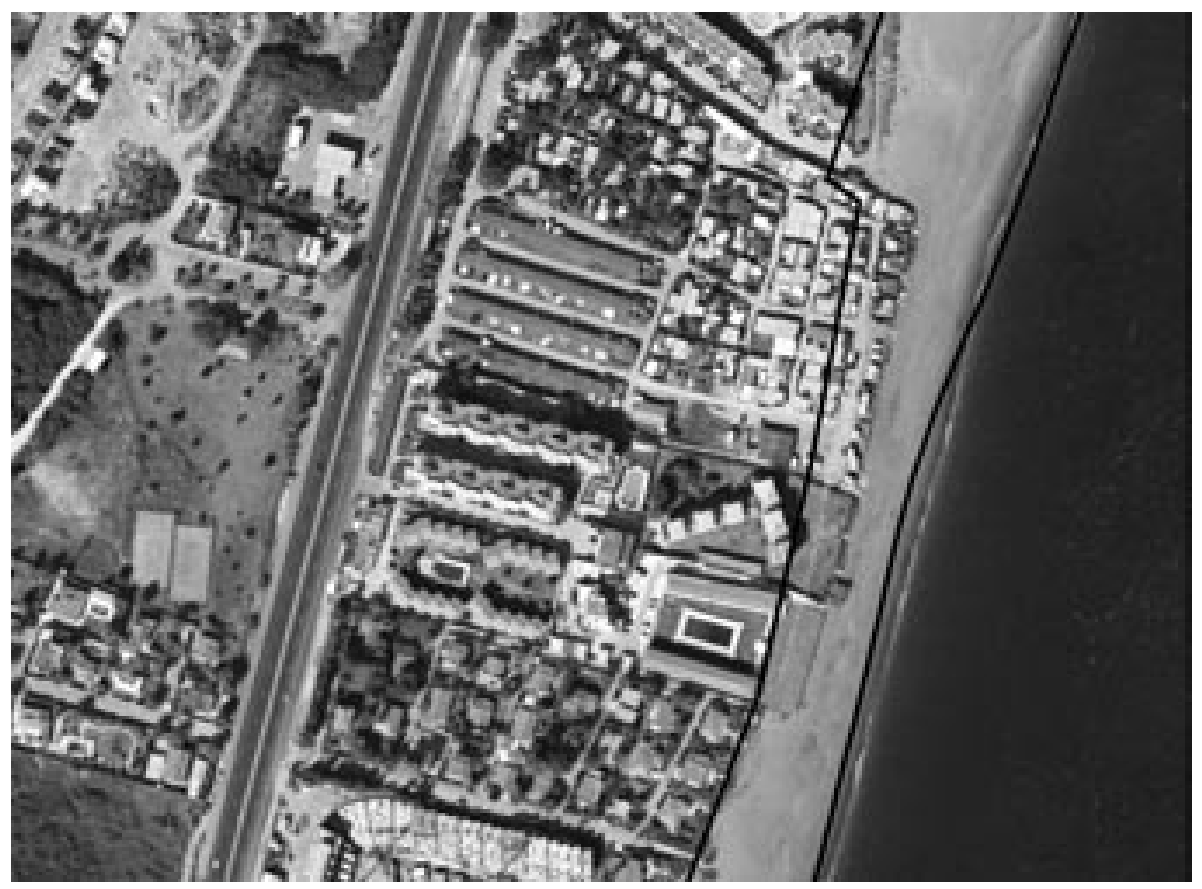

1. Transgesión del deslinde marítimo-terrestre por una urbanización en Manilva, construida con posterioridad a la entrada en vigor de la Ley de Costas. La línea negra marca la ocupación del dominio público marítimo-terrestre por las construcciones.

declarada Monumento Natural de Andalucía por el Decreto 250/2003, de 9 de septiembre, que le otorga protección especial. La situación paisajística y de conservación del monumento natural de las Dunas de Artola o Cabo Pino, en el término municipal de Marbella (Málaga), queda recogida en el tipo paisajístico de dunas y arenales en el Mapa de Paisajes de Andalucía, su localización en la Costa del Sol occidental ha convertido a este paraje de 19,27 ha en un enclave natural dentro de un entorno de urbanizaciones residenciales e instalaciones turísticas. Las Dunas de Artola son el último vestigio del sistema dunar de la costa malagueña que está caracterizado por tres tipos de dunas, separadas por valles interdunares. En cuanto a su aspecto físico, se puede distinguir: dunas fósiles en el interior con una vegetación compuesta por pinos y matorral mediterráneo, dunas móviles inactivas, en posición paralela a la costa en forma de barrera y, más cercanas al mar, dunas móviles activas, estás dos últimas cubiertas de comunidades vegetales psammófilas. Se añaden, además, otros elementos paisajísticos como el arroyo Cabrillas y la Torre de los Ladrones, torre defensiva declarada Bien de Interés Cultural. En consecuencia, este espacio muestra un paisaje residual que, por un lado, da el testimonio 
de la situación histórica de la costa malagueña y, por otro, pone de relieve la situación actual caracterizada por la presión urbanística residencial y turística de las zonas litorales.

Este enclave ha venido sufriendo durante los últimos años las presiones de los promotores inmobiliarios propietarios de los terrenos y el Ayuntamiento de Marbella interesados en urbanizar la zona. Autorizada la puesta en marcha de la urbanización de este enclave por el grupo GIL en junio de 1992 y sin la autorización de la Junta de Andalucía, se suscribió un convenio urbanístico entre los propietarios de los terrenos (varias sociedades anónimas de carácter efímero y difícil seguimiento legal) y el Ayuntamiento de Marbella el 8 de junio de 1992. Los responsables municipales y los dueños de los solares mantuvieron comunicaciones para que las obras comenzasen inmediatamente, dada la entrada en vigor de la moratoria prevista para el litoral en este año por la Ley de Ordenación del Territorio de Andalucía. La promoción inmobiliaria se levantaría en la zona de servidumbre marcada en el deslinde ya que las Dunas de Artola comprenden los límites del deslinde realizado por la Demarcación de Costas y ratificada por la Audiencia Nacional.

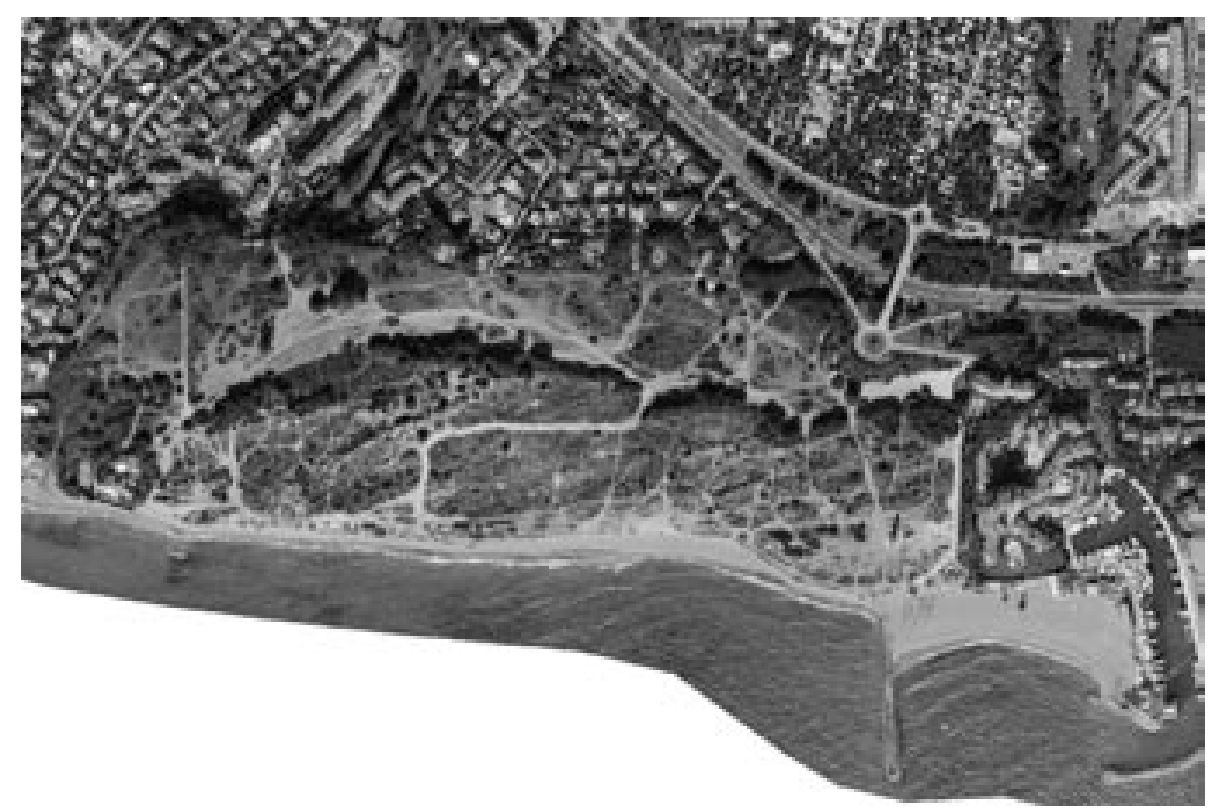

2. Dunas de Artola en el término municipal de Marbella. Declaradas Monumento Natural de Andalucía han estado varias veces a punto de ser urbanizadas. La presión urbanística sobre ellas es enorme. 


\section{CRITERIOS EMANADOS DEL GOBIERNO AUTONÓMICO}

Las políticas de Ordenación del Territorio llevadas a cabo a nivel autonómico han tenido como principal finalidad integrar, articular y compatibilizar espacialmente las intervenciones de las distintas administraciones públicas en el marco territorial andaluz. Sin embargo, este objetivo se ha conseguido en un buen grado a nivel autonómico, nacional y comunitario, pero escasamente a nivel local. Ha correspondido pues a la Administración Autonómica Andaluza formular propuestas de ordenación para el conjunto de su territorio ${ }^{15}$, sin perjuicio de la necesaria coordinación con los demás niveles políticos y administrativos. La oportunidad de esta iniciativa quedaba justificada por la implantación y consolidación del Estado de la Autonomías, que implicó la posibilidad y necesidad de implementar políticas de ordenación y desarrollo regional específicas, que solventarán o minimizaran los problemas concretos y propios de la escala regional, enfocando las iniciativas desde los intereses de la propia Comunidad Autónoma. La Carta Europea de Ordenación del Territorio, aprobada en Torremolinos en 1983 por la Conferencia de Ministros europeos responsables en esta materia, señalaba el nivel regional como un marco muy adecuado para la ordenación territorial. Desde un primer momento se han hecho propios en Andalucía los valores establecidos por la asunción de la Carta Europea de Ordenación del Territorio lo que significó la adopción de una serie de principios generales de indudable trascendencia posterior.

Una consecuencia de la aplicación de los principios de la Carta Europea de Ordenación del Territorio fue el proyecto de Esquema Europeo de Ordenación Territorial impulsado por el Consejo de Europa. Los informes previos de la Conferencia, en relación con dicha iniciativa, valoraron con fundamental la necesidad de completar los acercamientos desde los distintos niveles administrativos y donde el regional adquiría especial importancia. El protagonismo adquirido por los niveles regionales permitiría ir conformando el contenido y la imagen final del Esquema Europeo de Ordenación Territorial.

Desde la promulgación de la Constitución Española las administraciones autónomas en general, y la andaluza en particular, se habían acogido a la tradición europea de la realización de planes y programas regionales de desarrollo económico y territorial como método de actuación pública, dada la enorme dotación de fondos comunitarios para estas iniciativas. A partir de 1986, coincidiendo con el ingreso en la Comunidad Económica Europea, se produce un cierto cambio en la coyuntura económica que añade un factor más de oportunidad a las iniciativas

15. El Estatuto de Autonomía para Andalucía establece (Art. 13.8) de acuerdo con la Constitución Española (Art. 148.3) la competencia exclusiva de la Comunidad Autónoma en política territorial y ordenación del territorio. 
de planificación territorial. La política regional de la UE, dirigida a corregir los principales desequilibrios regionales existentes, ha venido dotando de cuantiosos fondos las iniciativas que fomentasen prioritariamente el desarrollo y el ajuste estructural de las regiones menos desarrolladas, así como la implementación de otros programas y políticas comunitarias de importantes consecuencias en el ámbito regional. Dichos programas comunitarios no sólo han venido aportando cuantiosos recursos económicos, sino que han tenido especial trascendencia en el propio proceso de planificación de la Comunidad Autónoma.

En el marco territorial, los principales conflictos o disfunciones actuales de la ordenación territorial en Andalucía han sido consecuencia de su historia reciente, y más concretamente de las orientaciones productivas que marcaron el crecimiento económico de los años sesenta y las posteriores readaptaciones que sufrió el aparato productivo regional durante los años de los crisis económica internacional de los setenta. De la primera etapa, se ha heredado como problema una sostenida tendencia hacia modelos territoriales fuertemente polarizados y ostensiblemente desequilibrados, así como la mayoría de las principales rupturas de los equilibrios ambientales de la región. De la segunda, de crisis y ajuste económico, además de los importantes problemas sociales, surgieron nuevos problemas territoriales derivados de los diferentes comportamientos económicos de las distintas áreas de la región.

A partir de 1986 se detectó un cambio de tendencia en sentido positivo que, evolucionó en el futuro acorde con las perspectivas de mayor integración económica con y en Europa y convergencia económica con los objetivos marcados por los acuerdos del Tratado de Maastricht en 1991. En concreto, la mayor integración económica con Europa ofreció a las políticas de ordenación del territorio la oportunidad de dar respuesta a tres retos: uno, aprovechar territorialmente las oportunidades derivadas de los nuevos procesos de desarrollo, basados tanto en la diversidad de experiencias de desarrollo endógeno en el medio rural, como en la tendencia a una mayor flexibilidad de localización de las nuevas actividades económicas; dos, orientar las fases de crecimiento económico con lo que se intentaba evitar la reproducción y profundización de modelos de ocupación territorialmente desequilibrados; y tres, superar los desequilibrios territoriales y ambientales heredados y evitar los derivados de las nuevas situaciones económicas.

A pesar de todo, hasta fechas recientes la gestión territorial y el desarrollo urbano han seguido caminos diferentes en Andalucía. Cuando el proceso de desarrollo de las últimas décadas ha originado graves disfuncionalidades en la organización del territorio de la región, la Ordenación del Territorio ha cobrado especial importancia como marco de actuación para definir el papel que cada área debe representar en el conjunto de la región. El sistema de los grandes núcleos de población o grandes ciudades ha asumido su responsabilidad en la estrategia 
de desarrollo socioeconómico de los distintos espacios. Entonces, el gobierno regional ha pasado a prestar especial importancia a los problemas de las grandes ciudades andaluzas como componentes del entramado funcional del sistema regional con la finalidad de articular un tejido urbano andaluz con el resto de España y Europa.

Pero este esfuerzo, entendido como un proceso de intervención sobre el territorio, ha requerido ineludiblemente la incorporación de nuevos instrumentos y figuras de planificación de cuya implantación ha surgido un nuevo marco de intervención en el territorio andaluz. El marco normativo e instrumental hasta fechas recientes ha presentado importantes deficiencias cuya resolución ha resultado indispensable para la actuación ordenada de las administraciones públicas en el territorio. La formación de las aglomeraciones urbanas y espacios turísticos litorales ha sido consecuencia de complejos procesos de ocupación del territorio, caracterizados por la superposición de múltiples actuaciones privadas, que han llevado a remolque a las actuaciones públicas. La suma de la planificación urbanística local y las políticas sectoriales de la Administración Estatal han configurado unos modelos territoriales carentes de una estrategia global o común de escala supralocal o supramunicipal, que otorgase al conjunto una mayor eficacia en su funcionamiento interno, identificase las potencialidades que se han derivado del proceso de desarrollo urbano y determinase los crecimientos e implantaciones de acuerdo a un estructura racional de respecto a los condiciones del medio ambiente como soporte físico de dichas instalaciones. Todo ello en claro incumplimiento de los postulados comunitarios en este sentido y a los que se habían venido acogiendo los distintos gobiernos nacionales.

La primera iniciativa autonómica de rango supramunicipal fueron las Directrices para la coordinación urbanística del Área Metropolitana de Sevilla. El proceso de coordinación urbanística metropolitana se inició con la Orden de 13 de Junio de 1984 del Consejero de Política Territorial por la que se acordó la formación de Directrices de Planeamiento Urbanístico para el área metropolitana de Sevilla. La iniciativa se inscribió en un programa de coordinación del planeamiento en ámbitos supramunicipales que tuvo escasa aplicación posterior. Los trabajos preparatorios empezaron en 1983 con la constitución en el seno de la Dirección General de Urbanismo de la Consejería de Política Territorial por aquel entonces, de un equipo técnico específicamente dedicado a la realización de los trabajos relativos al área de Sevilla. En Junio de 1984 fue presentado el documento: "Área Metropolitana de Sevilla. Propuesta para la Coordinación de las Políticas Urbanísticas Municipales". En él se señalaba explícitamente que el proceso iniciado se centraba en la coordinación y compatibilización del planeamiento urbanístico municipal como punto de arranque de un proceso general que incluiría a todas las administraciones urbanísticas o sectoriales que intervenían físicamente en dicho ámbito 
metropolitano. En Marzo de 1986 fue aprobado por la Comisión Provincial de Urbanismo de Sevilla, tras un largo proceso de trabajo conjunto de los órganos de la Consejería, los municipios y la Diputación Provincial, el Documento de Bases para la coordinación urbanística en el Área Metropolitana de Sevilla. Las Bases venían a cumplir un requerimiento necesario en el proceso de redacción de las Directrices como era el establecimiento de los criterios y objetivos de la política urbanística en dicho ámbito. Durante los años 1985 y 1987 se trabajó en el proceso de formulación de planeamiento general en los municipios del área. Cuando en 1986 se aprobaron las Bases, la situación de la política urbanística había evolucionado notablemente con respecto a la que se daba al comienzo del proceso de coordinación, habiéndose consolidado los procesos de coordinación interadministrativa en los ámbitos territoriales y sectoriales concretos. A principios de 1988, finalizados los procesos iniciados en 1985 de formulación de planeamiento, diseñados los elementos infraestructurales básico de interés metropolitano y en avanzado estado los trabajos de información y documentación necesarios, dio comienzo la redacción del documento de Directrices, que fue aprobado en Junio de 1989 (DCU, 1989).

Paralelamente a los primeros intentos de coordinar el planeamiento urbanístico en los espacios urbanos, la Consejería de Política Territorial (actualmente Consejería de Obras Públicas y Transportes) introdujo en su día el concepto de protección del medio físico en el panorama del planeamiento y la gestión del territorio. La formulación del programa de Planes Especiales de Protección del Medio Físico de ámbito provincial se inscribió en el desarrollo de las líneas de actuación trazadas por la Dirección General de Urbanismo en su documento programático de Septiembre de 1982. Y más concretamente en las que tenían por objetivos el fomento del planeamiento urbanístico y su integración en el sistema regional de planeamiento. Se buscaba que todas las líneas de actuación estuvieran conectadas e imbricadas entre sí, porque en definitiva todas participaban del objetivo central de la política urbanística regional por esas fechas: garantizar la efectividad y potenciar la operatividad del principio de intervención pública democrática en la dirección y control de los procesos territoriales y urbanos. La aplicación de la normativa de los planes especiales del medio físico en el ámbito provincial andaluz, aprobado por Orden del 6 de Marzo de 1987 del Consejero de Obras Públicas y Transportes, ante la ausencia de alguna otra figura o instrumento de planeamiento, ha sido uno de los principales valores de esta normativa. Así, estos documentos se constituyeron en instrumentos de regulación normativa a nivel supramunicipal, cuyas disposiciones debió incorporar el planeamiento urbanístico junto a los mecanismos propios que regulasen el uso y las actividades que se daban en el Suelo No Urbanizable que se afectase como protegido.

Los planes especiales del medio físico fueron unos documentos de excepcional valor, como se ha podido comprobar posteriormente. Gracias a ellos mu- 
chos espacios de gran valor ecológico y ambiental pudieron ser salvaguardados del proceso urbano. No obstante, debió ser revisado en su momento, ya que fue sufriendo progresivas modificaciones, desafecciones de espacios protegidos y correcciones de límites que le restaron vigencia. Si bien la no coincidencia temporal de vigencias entre los planeamientos municipales y los planes especiales hizo necesario el estudio comparativo de límites, aclarando dónde se contenían estas acepciones y las posibles zonas de conflicto entre ambos documentos, posteriormente se ha comprobado que esta posibilidad, contemplada en los mismos planes especiales, fue utilizada por las administraciones locales, a instancia de los grupos de presión económicos, como argumento para modificaciones de la protección y desafecciones, que fueron invalidando progresivamente los postulados de estos documentos. Estas incongruencias llevaron con el paso del tiempo a una situación de rechazo de los planes especiales de protección del medio físico por parte de los redactores del planeamiento urbanístico, esgrimiéndose múltiples argumentos para devaluar o intentar anular dichos documentos normativos.

Otro documento de grandes pretensiones y escasa repercusión fueron las Directrices Regionales del Litoral de Andalucía ${ }^{16}$. Por Decreto 76/1985, de 3 de Abril, se autorizaba la formulación de la que representaban una de las iniciativas de mayor rango y alcance llevadas a cabo por la Consejería de Obras Públicas y Transportes de la Junta de Andalucía y por Decreto 118/1990, de 17 de Abril, se aprobaban dichas Directrices, que afectaban a cinco provincias de las ocho andaluzas, e integraba a 71 municipios (la totalidad de los municipios litorales completos). Éstas suponían una iniciativa original respecto al ordenamiento existente para el litoral. Como se expresaba en el documento mismo, las Directrices del Litoral debían ser entendidas principalmente como un marco de referencia para el planeamiento urbanístico, que las debería desarrollar, y también para diversas políticas sectoriales que encontrarían en ellas objetivos y criterios ante las cuestiones suscitadas en relación al litoral. Las Directrices Regionales del Litoral de Andalucía contenían no sólo directrices propiamente dichas, sino también líneas de actuación, medidas y recomendaciones, en relación, por una parte a los recursos y actividades en este territorio y, por otra, a cada una de las unidades físicas en las que se catalogó y diferenciado el litoral (DRLA, 1990).

16. Como su propio nombre indica, el ámbito territorial de aplicación era litoral y el espacio marítimo proyectado desde éste, unos $2.281 \mathrm{~km}^{2}$ de aguas interiores, sobre cuya pesca se ejercían las competencias de ordenación y gestión de estos recursos, $13.935 \mathrm{~km}^{2}$ de mar territorial (esta delimitación, -doce millas náuticas a partir de las líneas de base recta- resulta impuesta por imperativos administrativos, obviando así la problemática delimitación de la plataforma continental) y aproximadamente $49.026 \mathrm{~km}^{2}$ de Zona Económica Exclusiva de cuyas aguas dependen importantes sectores productivos de la economía andaluza; en total $65.242 \mathrm{~km}^{2}$ lo que suponía el $75 \%$ de la superficie terrestre de la Comunidad Autónoma. 
Las disposiciones contenidas en las Directrices, según marcaba su artículo 1.1, tenían carácter vinculante para la actuación pública en cuanto a los objetivos y resultados que debían lograrse en el ámbito litoral, dejando los medios y forma concretos para conseguirlos a los distintos organismos administrativos, que deberían asegurar la coordinación de sus actuaciones para la más adecuada gestión del litoral y sus recursos; algo que nunca se llevó a cabo ya que las determinaciones de las Directrices venían a incidir en aspectos ya regulados por otras normas estatales, como son la Ley 29/1985, de Aguas, y la Ley 22/1988, de Costas, desarrollada por su Reglamento General Aprobado por Real Decreto 1471/1989, de 1 de Diciembre, con la intención de implementar la aplicación de las mismas al territorio andaluz. Por otra parte, ha de significarse que estas Directrices participaban del carácter de los actos preparatorios a que, en materia de planeamiento urbanístico, se hacían referencia en el art. 28 del Texto Refundido de la Ley sobre Régimen del Suelo y Ordenación Urbana de 1976 y art. 115 del Reglamento de Planeamiento.

Otro documento emanado del Consejo de Gobierno de la Junta de Andalucía en Acuerdo de 27 de Marzo de 1990 (BOJA 18/05/90, 1990), fueron las Bases para la Ordenación del Territorio de Andalucía, con carácter directivo ${ }^{17}$, que constituyeron un documento marco en el que se definían los objetivos, las estrategias y las principales actuaciones necesarias para la consecución de un modelo territorial, que propiciase un desarrollo económico equilibrado a medio y largo plazo. La primera justificación directa del documento provenía de la toma de conciencia de que la organización territorial la región por esas fechas no era satisfactoria y de que las necesidades de corrección del sistema territorial eran perentorias. La progresiva desarticulación de la región y en especial de ciertos espacios andaluces, las desigualdades en dotaciones sociales y calidad de vida, la polarización de la población y actividades económicas o productivas, el atraso estructural de muchas áreas, el consumo desordenado de recursos naturales y las rupturas de los equilibrios ambientales eran los principales problemas que ponían de manifiesto la necesidad de favorecer un modelo de organización territorial integrador, que apoyase la consecución del crecimiento económico y social y el equilibrio medioambiental como pilares básicos de la calidad de vida de los habitantes de la región (BASES, 1990).

En este contexto, se planteaba acometer, a escala regional, una serie de cuestiones, como se comprobó posteriormente, imposibles de abordar por sus dimensiones e implicaciones, por los marcos competenciales establecidos en esos momentos y por la ausencia de un marco normativo que permitiera llevarlas a cabo. Entre estas cuestiones destacamos las siguientes, por su

17. Ejercerían su influencia a través de la definición de criterios, objetivos, problemas y oportunidades de naturaleza territorial 
trascendencia para los espacios costeros: la distribución espacial, funciones y dotaciones de los asentamientos de población, las infraestructuras y la organización de las comunicaciones y transportes, la distribución y organización de las actividades económicas en el territorio y la utilización y regeneración de los recursos naturales. Realmente interesante era que las Bases plantearan en qué grado cada uno de estas cuestiones, en su configuración sobre el territorio, se presentaban como problema a resolver, como oportunidad a potenciar o como bien a preservar. No obstante, quizás los más interesante a nuestro entender, radica en que las Bases retomaban, aunque un poco tardíamente, la tarea de dotar al conjunto de la política regional de criterios y objetivos de carácter territorial, abriendo nuevas perspectivas y enfoques a los problemas que habían venido apareciendo en momentos especialmente delicados para Andalucía. El documento de las Bases, se situaba en una posición intermedia en el proceso de planificación territorial, en tanto que partían de las formulaciones existentes, para aproximarse progresivamente a un orden territorial general, sin agotar todas las posibilidades de definición de la política regional desde la perspectiva territorial.

Por consiguiente, éstas pretendían ejercer su influencia sobre el proceso de efectiva organización territorial en los siguientes sentidos: En primer lugar, las Bases fueron el primer paso en el camino de impulsar un proceso continuado para la consecución de un modelo de ordenación territorial a escala regional, a establecer mediante un plan regional de ordenación territorial. Es éste un objetivo a largo plazo, planteado desde hace bastante tiempo en muchas regiones europeas y ya abordado en España por algunas Comunidades Autónomas. En segundo lugar, recogían e integraban diversas iniciativas de planificación económica y sectorial. Esta integración de la planificación existente debería permitir un acercamiento real a la ordenación del territorio en términos de práctica política actual y de repercusión efectiva e inmediata. En tercer lugar, las Bases debían influir en las nuevas fórmulas de las políticas sectoriales con incidencia territorial regional, debiéndose convertir para ellas en un marco obligado de referencia; y en un horizonte general, para aumentar la coherencia de las futuras actuaciones departamentales. Y por último, en cuarto lugar, éstas ambicionaban constituirse como referente territorial imprescindible para la determinación de áreas de desarrollo integral, o de identificación de elementos espaciales que puedan convertirse en planes y programas operativos precisos.

Pues bien, todo lo planteado en las Bases quedaría supeditado a cuestiones mayores como la impuesta por la elevada desarticulación del sistema urbano andaluz a escala regional que repercutía en una clara potenciación de las relaciones y el nivel organizativo regional. Estas relaciones, en el contexto andaluz, se mostraran ligadas fundamentalmente a actividades de consumo 
para las que los centros subregionales (siete de los nueve centros considerados como subregionales -aparte del papel de Sevilla como centro subregional- son capitales provinciales y sus ámbitos correspondan, de modo muy general, a los de los ámbitos provinciales), se convertían en proveedores de bienes y servicios de nivel superior. En este contexto, donde la organización del sistema urbano andaluz presenta en sus escalones superiores una estructura regional con un centro que no logra articular al conjunto del territorio, la organización subregional aparecerá claramente fortalecida. Este fortalecimiento será interno a cada uno de los espacios subregionales o provinciales, ya que las relaciones de los centros con otros espacios seguirían siendo escasas. Ha sido necesario incidir en esta configuración, aunque someramente, para resaltar la importancia que posteriormente tendrá ésta en la definición de los ámbitos territoriales que se adscribirán a los distintos planes subregionales.

Ante las situaciones que se venían presentando desde la perspectiva territorial y para apoyar las actuaciones en materia de Ordenación del Territorio del Gobierno Autonómico se dotó a la Comunidad de una normativa en esta materia con la Ley 1/1994, de 11 de Enero, de Ordenación del Territorio de la Comunidad Autónoma de Andalucía (BOJA numero 8, de 22 de enero de 1994). Con anterioridad a la fecha de promulgación de la Ley de Ordenación del Territorio de Andalucía, se habían estado llevando a cabo durante los diez primeros años de autogobierno en la región andaluza una serie de proyectos territoriales y regionales sin sustento legal que los pudiese hacer realidad, entre otros, las mencionadas Bases para la Ordenación del Territorio de Andalucía, aprobadas el 27 de marzo de 1990 por el Consejo de Gobierno de la Junta de Andalucía y las Directrices para la Coordinación Urbanística del Área Metropolitana de Sevilla, aprobadas en junio de 1989.

Esta Ley deriva del artículo 148.1.3 ${ }^{\mathrm{a}}$ de la Constitución, en virtud del cual las Comunidades Autónomas pueden asumir competencias en ordenación del territorio, urbanismo y vivienda, y se dicta con base en la competencia exclusiva que, en dicha materia, atribuye a la Comunidad de Andalucía el artículo 13.8 de su Estatuto de Autonomía. Ha seguido etapas análogas a las discurridas por las disposiciones que, con fines similares, han dictado otras comunidades autónomas.

El análisis de esta Ley evidencia unas disposiciones claras y sencillas, ordenadas de acuerdo a sus fines, habiendo huido de introducir denominaciones alejadas de las ya clásicas. Sus objetivos específicos, como se enuncia en su exposición de motivos, son la ordenación del territorio y la articulación territorial interna y con el exterior de la Comunidad, así como la distribución de las actividades y de los usos del suelo, armonizada con el desarrollo equilibrado, las potencialidades del territorio y la protección de la naturaleza y del patrimonio histórico. Para ello, la Ley creaba dos instrumentos de ordenación 
integral: el Plan de Ordenación del Territorio de Andalucía y los Planes de Ordenación del Territorio de Ámbito Subregional, a la vez que establecía las actividades de planificación que tendrían la consideración de planes con incidencia en esta materia. Para el primero de ellos la Ley fijaba su tramitación hasta su promulgación, estando prevista la elaboración de bases o estrategias regionales, como verdaderos avances preparatorios del Plan. Los segundos, se correspondían con territorios, partes del de toda la Comunidad, que precisaban la mejora de sus estructuras y podían constituir ámbitos funcionales unitarios, posibilitando su adaptación a las circunstancias que se presentasen en los mismos, en relación al interés supramunicipal de los espacios que sirviesen y sin que pudieran establecerse clasificaciones del suelo. Su función básica radicaba en establecer los elementos básicos para la organización y estructura del territorio al que se circunscriben, siendo marco de referencia obligado para el desarrollo de la política territorial de las administraciones y entidades públicas y para las actividades de la iniciativa privada. Así, de los dos instrumentos establecidos por Ley de Ordenación del Territorio para la planificación integral, se ha desarrollado sólo uno, el de los Planes de Ordenación del Territorio de ámbito subregional.

El Plan de Ordenación del Territorio de Andalucía, en su formulación, constituye una decisión del Gobierno Autónomo (Decreto 83/1995, de 28 de marzo) que debe entenderse en relación con sus antecedentes y con el conjunto de las políticas de desarrollo regional. El Decreto 103/1999, de 4 de mayo aprueba las Bases y Estrategias del Plan de Ordenación del Territorio de Andalucía. El Consejo de Gobierno, por Decreto 83/1995, de 28 de marzo, acordó formular el Plan de Ordenación del Territorio de Andalucía, en cuyo artículo 8 , apartado 1, como primera fase del proceso de redacción la elaboración de un documento de Bases y Estrategias con el alcance previsto en el artículo 9 de la Ley 1/94, de 11 de enero, de Ordenación del Territorio de la Comunidad Autónoma de Andalucía. Su finalidad ha sido la de orientar en los contenidos y propuestas del Plan, estableciendo el diagnóstico de las oportunidades y problemas regionales así como sus objetivos y estrategias de desarrollo. La elaboración de dicho documento de Bases y Estrategias se ha realizado en los términos establecidos en la Ley 1/94, y de acuerdo con las disposiciones del Decreto 83/1995, de 28 de marzo, tanto en sus aspectos procedimentales como de contenido sustantivo y material.

Por otra parte, la Administración Autonómica está intentando llevar a cabo la ordenación territorial del espacio litoral mediante Planes de Ordenación del Territorio de ámbito subregional que incluyen a las distintas zonas del litoral andaluz. En la actualidad se encuentran aprobados 5 planes: Ámbito de Doñana (Huelva), Bahía de Cádiz (Cádiz), Poniente de Almería (Almería); Costa del Sol Occidental (Málaga) y Litoral Oriental-Axarquía (Málaga); 1 se encuentra en tramitación: Litoral Occidental de Huelva (Huelva); 5 en redac- 
ción: Costa Noroeste de Cádiz (Cádiz), La Janda (Cádiz), Campo de Gibraltar (Cádiz), Aglomeración Urbana de Málaga (Málaga) y Levante de Almería (Almería); y 3 planes en próxima formulación: Litoral de Granada (Granada), Aglomeración Urbana de Huelva (Huelva) y Aglomeración Urbana de Almería (Almería), con lo que en total se dispone de 14 planes de ordenación del territorio de ámbito subregional para el litoral andaluz.

Los Planes de Ordenación del Territorio de ámbito subregional mencionados abordan el análisis territorial de los municipios incluidos en cada ámbito desde una perspectiva amplia que incluye, expresado sintéticamente: por una parte, el esquema territorial de las infraestructuras básicas, distribución de los equipamientos y servicios de carácter supramunicipal, con el objetivo de mejorar las articulación territorial y garantizar la accesibilidad y funcionalidad del territorio acorde con el nivel de actividad e intensidad de las relaciones existentes entre los asentamientos que integran cada ámbito; y, por otra, la indicación de las zonas para la ordenación y compatibilización de los usos del territorio y para la protección y mejora del paisaje, de los recursos naturales y del patrimonio histórico y cultural, estableciendo los criterios y las medidas que hayan de ser desarrolladas por los distintos órganos de las Administraciones Públicas, con ello se pretende preservar los espacios con valores medioambientales, paisajísticos e histórico culturales, garantizando el aprovechamiento de las potencialidades productivas del territorio. Un aspecto muy importante de estos planes es que las determinaciones de los Planes con incidencia en la Ordenación del Territorio y los Planes Urbanísticos deberán adaptarse a ellos.

Otro documento con pretendida incidencia en la gestión territorial del ámbito litoral andaluz han sido el Plan de Mejora Ambiental del Litoral emanado del Plan de Medio Ambiente de Andalucía 1997-2002 que ha tenido una incidencia escasa sobre el litoral andaluz en lo referente a la mejora y ordenación del medio natural costero, con leves avances en la restauración del espacio litoral en sí, habiéndose centrando casi en exclusiva en el control de la calidad de las aguas y por tanto, en la puesta en marcha de planes de corrección de vertidos.

Con cierto interés se mira el Proyecto de Medidas para el Desarrollo del Pacto por la Defensa del Litoral Andaluz, promovido por la Consejería de Turismo y Deporte de la Junta de Andalucía (enero de 2004), que ha pretendido establecer una estrategia económico-territorial para el cambio del modelo turístico actual. Este documento proviene de un documento denominado "Bases para un Pacto por la Defensa del Litoral Andaluz" que fue aprobado por el Pleno del Consejo Andaluz de Turismo, el día 10 de julio de 2003, que tenía como objetivo efectuar un diagnóstico global y compartido por los principales agentes que inciden en el desarrollo turístico del litoral andaluz, en el que se 
exponía la necesidad de articular las políticas turísticas y territoriales, bajo el principio de la sostenibilidad. No obstante, según los postulados vertidos en este documento y para que fuese efectivo desde el punto de vista territorial, no sólo de la actividad económicas en sí misma, deberían definirse las responsabilidades y el ámbito competencial de los distintos agentes implicados en la aplicación ya que se pretenden llevar a cabo medidas que afectan a distintos instrumentos normativos y de planificación que tienen incidencia en el litoral. De otro modo, no se podrá llevar a cabo una actuación coordinada entre los mismos.

En definitiva, la intervención planificadora llevada a cabo por el Gobierno Autonómico ha buscado la ordenación de los usos del suelo en el territorio andaluz, acometiendo la resolución de los conflictos territoriales no abordables desde la óptica municipal e intentando dotar al ámbito litoral de un marco normativo de ordenación territorial que permita reducir las incertidumbres existentes y establecer las medidas de actuación necesarias para minimizar los grandes problemas generados por la expansión urbana, no olvidando el desarrollo de las enormes potencialidades del litoral.

\section{CRITERIOS EMANADOS DE LAS ADMINISTRACIONES LOCALES}

El papel de la administración local ha sido y es fundamental en la ocupación de los espacios litorales de Andalucía. De aplicación directa son los criterios emanados desde los ayuntamientos (criterios elaborados junto con otras partes implicadas directamente, como las empresas, agentes económicos y habitantes de la zona). Las estrategias adoptadas desde otros niveles no tienen validez si no son aplicadas o vigiladas en su aplicación por las autoridades a nivel local. El Gobierno Regional puede orientar la coordinación de iniciativas locales, proporcionando el marco jurídico e institucional necesario para facilitar las medidas locales. La estrategia para la gestión integrada de las zonas costeras propuesta por la UE tendrá éxito si es asumida a nivel local, a través de actuación armoniosa entre las distintas esferas administrativas. En muchos casos se requiere la cooperación intermunicipal. Por ejemplo, sería más lógico que los municipios que comparten litoral coordinen sus actividades en lugar de adoptar medidas locales que a veces entran en conflicto con sus vecinos más inmediatos.

La planificación y gestión urbanística a nivel local es un tema candente, siempre lo ha sido, dado que la intensidad del proceso de urbanización permite caracterizar un modelo de ocupación que configura el espacio costero centrado en su capacidad residencial. No parecen existir límites para el desarrollo 
urbanístico del litoral, tendente a la masificación constructiva y con graves impactos ambientales. Es fácil comprobar que una misma zona costera puede ser objeto de distintas formas de actuar respecto al tratamiento del litoral con dependencia sólo y exclusivamente de los límites administrativos municipales. Encontramos medidas inconexas y postulados urbanísticos distintos y no coordinados sobre espacios con las mismas características naturales. Muchas veces los ayuntamientos se acusan unos a otros de contaminar la calidad de sus aguas costeras y sus playas por el mal funcionamiento de sus propias plantas de depuración de aguas residuales debido a que las infraestructuras no son capaces de dar servicio a una demanda para la que no fueron concebidas. Claro ejemplo de una situación que en muchos momentos ha venido rondando lo caótico son los procesos de formulación de planeamiento de los municipios del litoral y las disparidades en sus criterios y objetivos de ordenación ${ }^{18}$.

\section{PRINCIPALES DESAFÍOS DE FUTURO}

A la vista de la situación actual cuesta creer que la percepción de los problemas ambientales del litoral se haya extendido a los agentes económicos y a algunos ámbitos de las administraciones públicas. En relación con la percepción de la problemática litoral asociada al desarrollo económico parece muy difícil que en la actualidad se puedan arbitrar medidas que pudieran limitar el crecimiento urbanístico para evitar la total ocupación del frente litoral y el deterioro de los espacios naturales y litorales, protegidos o no.

En los últimos decenios el litoral andaluz ha estado sometido a una urbanización cada vez más intensa. Los proyectos inmobiliarios al albor del furor urbanístico, con demasiada frecuencia no han tenido ni orden ni concierto. Una de las causas de la expansión urbanística descontrolada ha sido la proliferación de segundas residencias, muchas de las cuales permanecen vacías la mayor parte del año y sólo se ocupan durante períodos concretos. Sin embargo, son culpables con frecuencia de la destrucción hábitats naturales frágiles e impiden el acceso público a playas y parajes naturales. El problema de la edificación excesiva de la costa es particularmente acusado en algunas zonas del litoral, donde muchas urbanizaciones turísticas y segundas residencias surgieron de modo ilegal, aunque posteriormente fueron legalizadas, y otras

18. Para profundizar más en este asunto se puede consultar: GALACHO, F.B. (2005): "El planeamiento urbanístico municipal de la Costa del Sol. Procesos de formulación y criterios de ordenación". Baetica. Estudios de Arte, Geografía e Historia, 27, Facultad de Filosofía y Letras, Universidad de Málaga, 97-114. 
incumplen las normas más elementales de ordenación territorial. El modelo de desarrollo urbanístico predominante en el litoral es el de la urbanización dispersa e inconexa en el territorio, que finalmente da lugar a un continuo urbano desarticulado y desequilibrado por la unión de piezas inconexas que son cosidas por unas infraestructuras improvisadas.

El trazado de las infraestructuras viarias en el litoral se ha desarrollado de modo poco prudente y en algunos casos con importantes repercusiones en la frágil dinámica costera y en el paisaje. El desorden manifestado para la expansión urbana, también se ha extendido a las redes de transporte que igualmente han venido siendo objeto de una mala planificación. El tránsito costero ha venido planteando problemas especialmente complejos limitando o perjudicando los desplazamientos. Sin conexiones adecuadas, unas redes de transporte erróneamente concebidas, sólo en función del flujo de turistas, están provocando problemas de contaminación y congestión, así como la destrucción de hábitats. Por tanto, es posible afirmar que el equilibrio entre accesibilidad y protección del medio ambiente rara vez se encuentran y el diseño de las infraestructuras ha concedido poca atención a las características específicas de las zonas costeras.

Las zonas costeras andaluzas sufren otras amenazas: la contaminación provocada por los vertidos marítimos y la de origen terrestre. Sobre ellas no sólo se cierne las amenazas de contaminación por catástrofes marítimas tales como mareas negras o vertidos de productos químicos, sino también se ven afectadas por la contaminación que se genera en la línea terrestre de costa. Uno de los problemas más graves para la calidad ambiental de la costa andaluza son los vertidos, accidentales o intencionados, de sustancias contaminantes en las aguas litorales. La calidad de las aguas litorales y de los ecosistemas marinos se han visto netamente afectadas como consecuencias de los continuos vertidos de sustancias contaminantes durante los últimos años. Muestra de ello son los episodios de contaminación marina que se producen en el Estrecho de Gibraltar y en la Bahía de Algeciras por vertidos incontrolados o limpieza de fondos de los petroleros que utilizan la gran vía marítima del Estrecho de Gibraltar. La preocupación social generada por estos incidentes no parece afectar a los causantes o responsables de estos vertidos y solo la constancia de las administraciones puede evitar episodios de desastres ecológicos de gran magnitud como los producidos recientemente en otras zonas litorales.

El tratamiento de las aguas residuales es una tarea sobre la que hay que trabajar todavía mucho. El Plan de Mejora Ambiental del Litoral dentro del marco del Plan de Medio Ambiente de Andalucía 1997-2002 se ha centrado en el control de la calidad de las aguas y por tanto, en la puesta en marcha de planes de corrección de vertidos. Sin embargo, las infraestructuras de saneamiento y depuración de aguas residuales urbanas de los municipios han veni- 
do funcionando la límite de su capacidad, dado el desfase entre construcción de vivienda e inversión en redes de saneamiento o instalaciones de tratamiento de aguas residuales, lo que ha producido vertidos de aguas residuales urbanas con escaso grado de depuración y simplemente con tratamientos primarios en contra de lo que obliga la Directiva 91/271. Como ejemplos, podemos citar, el caso de la Bahía de Algeciras que carece de estaciones de depuración de aguas residuales con tratamiento secundario y el litoral oriental de la provincia de Málaga que carece de red de saneamiento integral en muchos tramos y en el que se producen aún en la actualidad vertidos directos de aguas residuales a los cauces y arroyos o directamente a la playa desde las urbanizaciones residenciales. En el mismo caso que la Bahía de Algeciras se encuentra la zona de Torrox-Nerja donde está pendiente la construcción de una E.D.A.R. Todo ello se traduce en que las condiciones higiénico-sanitarias de algunas playas sean muy deficientes.

Otro aspecto importante es que en el litoral andaluz se encuentran algunos de los hábitats naturales más ricos y frágiles de la Unión Europea. Está a la orden del día la presión sobre los hábitats naturales del litoral, cuando no su eliminación, con la consiguiente pérdida de biodiversidad. El crecimiento demográfico y la evolución de las actividades económicas están provocando alteraciones en el lecho marino y en playas, estuarios y riberas. La expansión urbanística ha sido la responsable de la destrucción de hábitats costeros de gran importancia, especialmente humedales, marismas, dunas y acantilados que albergaban muchas especies vegetales y animales. Esta pérdida de hábitats no afecta sólo a la biodiversidad sino que repercute negativamente sobre los recursos hídricos y la erosión del litoral; ejemplo de ello ha sido el retroceso experimentado por numerosas playas del litoral, principalmente aquellas más cercanas a las zonas más dinámicas de ocupación urbanística. A pesar de todo, algunos planificadores a nivel local no consideran, al parecer, que la destrucción de hábitats costeros sea un problema. Las reivindicaciones a favor de la protección de esos hábitats naturales se consideran demasiado restrictivas a los ojos de las autoridades locales deseosas de impulsar la actividad económica a través del urbanismo mediante la construcción de más viviendas, más carreteras, más complejos turísticos y más establecimientos comerciales. No siendo conscientes de que la destrucción de hábitats es perjudicial también para las economías de los municipios costeros. La pérdida de espacios naturales de gran valor ecológico y estético impide también a las localidades costeras desarrollar, por ejemplo, el turismo ecológico y otras muchas actividades recreativas al aire libre, que sin embargo, publicitan en sus propias páginas Web, a modo de reclamo turístico.

Como se sabe, los hábitats naturales influyen poderosamente en la calidad de vida de los ciudadanos. Estas zonas les ofrecen diversas posibilidades. 
Según lo observado sobre el territorio litoral no parece que estos espacios puedan convertirse en un rasgo distintivo o apreciado de la zona urbana que se está configurando. La mayoría de los hábitats naturales con valor ecológico están protegidos pero sufren grandes presiones que atentan contra su biodiversidad y su riqueza ecológica. Paradójicamente el urbanismo desarrollado en el litoral no plantea proteger de la urbanización los espacios protegidos, y en medida alguna, se han llevado a cabo planteamientos de incorporar estos espacios en sus postulados.

La presión es elevada sobre los recursos naturales, en especial: sobre el agua, necesaria para el desarrollo de las actividades urbanas y agrícolas. En el litoral se originan problemas de sobreexplotación y deterioro de la calidad de los acuíferos costeros.

Como se puede entender según lo expuesto hasta aquí, la necesidad de una estrategia costera para el litoral andaluz es apremiante. Es necesario aplicar un planteamiento coordinado en la región a la política de costas para que los problemas que se han expuesto anteriormente no empeoren. Las tendencias de futuro apuntan que la población de las zonas costeras y que utilizan recursos costeros va a seguir aumentando en un futuro previsible. Si no se toman medidas para gestionar las cada vez más numerosas presiones que se ejercen sobre el litoral, problemas tales como la desaparición de hábitats, la contaminación, la masificación urbanística van a terminar destruyendo algunas de las zonas más bellas, con mayor biodiversidad y más frágiles del litoral, mientras las comunidades costeras sufren una pérdida de valor importante pareja a la desaparición de recursos de gran valor económico. 


\section{BIBLIOGRAFÍA}

BASES (1990): Bases para la Ordenación del Territorio de Andalucía. Consejería de Obras Públicas y Transportes, Junta de Andalucía, Sevilla. 195 pp.

CARAVACA, I. y ZOIDO, F. (2001): "Cambios socioeconómicos, industria y desarrollo territorial en Andalucía”, Publicado en Prensa. Tomado de la dirección Web: http://www.ues.es/giest/art_zoido-inmaculada.htm

COM (2004) 60 final: Hacia una estrategia temática sobre el medio ambiente urbano, Comunicación de la Comisión al Consejo, al Parlamento Europeo, al Comité Económico y Social Europeo y al Comité de las Regiones. Comisión de las Comunidades Europeas, Bruselas, 2004. http://europa.eu.net

DCU (1989): Directrices para la Coordinación Urbanística. Consejería de Obras Públicas y Transportes, Junta de Andalucía, Sevilla. $250 \mathrm{pp}$.

DRLA (1990): Directrices Regionales del litoral de Andalucía, Consejería de Obras Públicas y Transportes, Junta de Andalucía, Sevilla. 172 pp.

GALACHO, F.B. y LARRUBIA, R. (2002): Usos del suelo y territorio. Análisis de la situación ambiental de la provincia de Málaga. Oficina Técnica MADECA10 (Diputación Provincial de Málaga), Málaga, 2002. 91 pp. y Anexo Cartográfico. Disponible en la dirección Web: http://www.madeca10.info/component/ option,com_remository/Itemid,55/func,selectfolder/filecatid,4/

MMA -Ministerio de Medio Ambiente- (2004): Actuaciones Públicas en Materia de Medio Ambiente, Ministerio de Medio Ambiente, Madrid, www.mma.es

PEOT -Perspectiva Europea de Ordenación Territorial- (1999): Towards Balanced and Sustainable Development of the Territory of the European Union, Official Publications of the European Communities, Bruselas, 1999, 87 pp.

UE -Unión Europea- (2001): La Unión Europea apuesta por las zonas costeras. Un cambio de rumbo para las zonas costeras europeas, Comisión Europea, Dirección General de Medio Ambiente, Oficina de Publicaciones Oficiales de las Comunidades Europeas, Luxemburgo, http://europa.eu.net 